\title{
A NON-NOETHERIAN FACTORIAL RING $\left({ }^{1}\right)$
}

\author{
$\mathrm{BY}$
}

\section{JOHN DAVID}

\begin{abstract}
This paper supplies a counterexample to the conjecture that factorial implies Noetherian in finite Krull dimension. The example is the inte gral closure of a three-dimensional Noetherian ring, and is the union of Noetherian domains, which are proven to be factorial by means of derivation techniques.
\end{abstract}

0 . Introduction. This paper touches on the previously unexplored problem of when the factorial property implies the Noetherian property, in the category of commutative domains with unit.

Due to the abundant existence of non-Noetherian factorial rings in infinite Krull dimension, one restricts one's attention to rings of finite Krull dimension. However, this paper shows that one must make distinctions even finer than that of Krull dimension, finite or infinite, to properly treat factorial implies Noetherian. That is, there is a non -Noetherian factorial ring in dimension three.

1. Notation. We will retain the following notation for the remainder of the paper.

(i) "Dimension" means Krull dimension.

(ii) $K$ is a field of characteristic 2 such that $\left[K: K^{2}\right]$ is countably infinite. $\left\{b_{1}, c_{1}, \cdots, b_{k}, c_{k}, \cdots\right\}$ is a 2-base for $K$ over $K^{2}$.

(iii) $R^{*}=K[[x, y, z]], R=K^{2}[[x, y, z]][K]$ where $x, y, z$ are algebraically independent variables over $K$.

(iv) $d=\sum_{i=1}^{\infty} b_{i} y x^{i}+\sum_{i=1}^{\infty} c_{i} z x^{i}$.

For $N=1,2, \cdots$,

$$
d_{N}=\sum_{i=0}^{\infty} b_{i+N} y x^{i}+\sum_{i=0}^{\infty} c_{i+N} z x^{i}, \quad e_{N}=\sum_{i=0}^{\infty} b_{i+N} x^{i}, \quad f_{N}=\sum_{i=0}^{\infty} c_{i+N} x^{i} .
$$

For $N \neq 1, a_{N}=\sum_{i=1}^{N-1} b_{i} y x^{i}+\sum_{i=1}^{N-1} c_{i} z x^{i}, \alpha_{1}=0$.

(v) $T$ = the integral closure of $R[d]$ in its quotient field.

Received by the editors October 12, 1971.

AMS 1970 subject classifications. Primary 13F 15; Secondary 13E05, 13B20, 17 B 40.

Key words and phrases. Factorial, Krull dimension, Noetherian, derivation, integral closure.

(1) This work was done while the author was an NSF trainee. It will form part of his doctoral dissertation at the University of Rochester. He wishes to thank William Heinzer for his help. 
(vi) $H_{N}=K^{2}\left[b_{1}, c_{1}, \cdots, b_{N-1}, c_{N-1}\right][[x, y, z]]\left[e_{N}, f_{N}\right]$,

$K_{N}=$ the quotient field of $H_{N}$,

$I_{N}=K^{2}\left[b_{1}, c_{1}, \cdots, b_{N-1}, c_{N-1} 1[[x, y, z]]\left[d_{N}\right]\right.$,

$L_{N}=$ the quotient field of $I_{N}$,

$S_{N}=K^{2}\left[b_{1}, c_{1}, \cdots, b_{N-1}, c_{N-1}\right][[x, y, z]]$.

\section{Some theorems by M. Nagata and P. Samuel.}

Theorem 1 (Nagata). $(R,(x, y, z))$ is a regular local Zariski ring, $R^{*}$ the completion of $R$ and $\sum_{i, j, k} a_{i j k} x^{i} y^{j} z^{k} \in R$ iff $\left\{a_{i j k}\right\}$ belongs to a finite field extension of $K^{2}$.

Proof. See [1, p.206].

Theorem 2 (Nagata). $T$ is a tbree Krull-dimensional, non-Noetberian local ring with maximal ideal $(x, y, z)$.

Proof. See [1, p.208].

Theorem 3 (Samuel). Let $A$ be a UFD of characteristic $p \neq 0, L$ its quotient field, $\Delta$ a derivation of $L$ such that $\Delta(A) \subseteq A, L^{\prime}=\operatorname{Ker}(\Delta)$, and $A^{\prime}$ the Krull ring $L^{\prime} \cap A$. Define the logarithmic derivatives, $D$, of $\Delta$ relative to $A$ as the additive subgroup of $A$ consisting of elements of the form $\Delta t / t, t \in L$. The logarithmic derivatives of unity, $D^{\prime}$, are defined to be the subgroup of $D$ consisting of those elements that can be written as $\Delta u / u$ where $u$ is a unit of $A$. Then if $D=D^{\prime}, A^{\prime}$ is a UFD.

Proof. See [2, p.86].

Lemma A (Samuel). Let $L$ be a field of characteristic $p \neq 0, \Delta$ a derivation of $L, L^{\prime}$ the subfield $\operatorname{Ker}(\Delta)$. If $\left[L: L^{\prime}\right]=p$, then there exists $a \in L^{\prime}$ sucb that $\Delta^{p}=a \Delta .\left(\Delta^{p}\right.$ is $\Delta$ composed with itself $p$ times.)

Proof. See [2, p.87].

Lemma B (Samuel). With the same notation and bypotbeses in the above theorem and lemma, so that an element $t$ of $A$ is a logarithmic derivative of $\Delta$ with respect to $A$, it is necessary and sufficient that $\Delta^{p-1}(t)=a t-t^{p}$.

Proof. See [2, p.88].

3. $T$ is a Krull ring which is a union of an ascending chain of Noetherian three dimensional nonregular UFD's.

Proposition 1. T is a Krull ring.

Proof. This follows from 33.10 of $[1]$, as $R[d]$ is a Noetherian integral domain. 
Lemma 2.1. $W=R\left[e_{1}, f_{1}, \cdots, e_{k}, f_{k}, \cdots\right]$ is normal.

Proof. Let $g \in W^{\prime}$, the derived normal ring of $W$. Then $\exists p, q, r, s, t \in R$ such that $g=\left(p+q e_{n}+r f_{n}+s e_{n} f_{n}\right) / t$ for some $n$ as $R\left[e_{1}, f_{1}, \cdots, e_{l}, f_{l}\right]=$ $R\left[e_{l}, f_{l}\right]$ and the squares of elements of $R^{*}$ lie in $R$. As the coefficients of the terms of $p, q, r, s$ and $t$ together generate a finite extension of $K^{2}$ and because

$$
\begin{array}{cl}
e_{n}=b_{n}+b_{n+1} x+\cdots+b_{m-1} x^{m-n-1}+x^{m-n} e_{m^{\prime}} & m \geq n, \\
f_{n}=c_{n}+c_{n+1} x+\cdots+c_{m-1} x^{m-n-1}+x^{m-n} f_{m}, & m \geq n,
\end{array}
$$

we get $g$ contained in the derived normal ring of $H_{N}$ for some $N$, as $g^{2} \epsilon$ $K^{2}[[x, y, z]]$.

$g \in W$ follows from the next lemma and ${ }_{N} \subseteq W$.

Lemma 2.2. $H_{N}$ is a regular local ring.

Proof. Since $H_{N}$ is a finite module extension if $K^{2}[[x, y, z]]$, it is three dimensional local.

We thus need only show its maximal ideal, $m$, is $(x, y, z)$. Since $H_{N} \subseteq R^{*}$, an element of $H_{N}$ is a nonunit if it has subdegree $\geq 1$. The converse is also true since it is true of $K^{2}[[x, y, z]]$, and the squares of elements of $H_{N}$ lie in $K^{2}[[x, y, z]]$ and units of $H_{N}$ are such iff their squares are. Now let $a \in m$. Thus $a^{2} \in(x, y, z) K^{2}[[x, y, z]]$. Thus, as a power series in $R^{*}$, a must be of subdegree one or greater. Thus

$$
\alpha=k_{0}+k_{1} s_{1}+k_{2} s_{2}+\cdots+k_{q} s_{q}
$$

where $s_{i}$ are various products of $b_{i}$ 's, $c_{i}$ 's $(i<N), e_{N}$ and $f_{N}$ that are squarefree, and $k_{j} \epsilon K^{2}[[x, y, z]]$.

Since the zero degree forms of the $s_{i}$ are linearly independent over $K^{2}$, the subdegree of $\alpha$ is $\geq 1$ iff the subdegree of each of the $k_{i}$ 's are $\geq 1$, iff $k_{i} \epsilon$ $(x, y, z) K^{2}[[x, y, z]]$.

We conclude $m=(x, y, z) H_{N}$.

Lemma 2.3. 1, $e_{N}, f_{N}, e_{N} f_{N}$ are linearly independent over the quotient field of $R$.

Proof. It suffices to check independence over $R$. Let $r_{i} \in R$ such that

$$
r_{1}+r_{2} e_{N}+r_{3} f_{N}+r_{4} e_{N} f_{N}=0 .
$$

$\exists M>0$ such that $r_{i} \in S_{M} \forall i$. Let $Q=\max \{N, M\}$. Then

$$
\begin{aligned}
& e_{N}=b_{N}+b_{N+1} x+\cdots+b_{Q-1} x^{Q-N-1}+x^{Q-N} e_{Q}, \\
& f_{N}=c_{N}+c_{N+1} x+\cdots+c_{Q-1} x^{Q-N-1}+x^{Q-N} f_{Q} .
\end{aligned}
$$


Substituting these two equations into $(*)$, we have a relation of the form

$$
r_{1}^{\prime}+r_{2}^{\prime} e_{Q}+r_{3}^{\prime}+r_{4}^{\prime} e_{Q} f_{Q}=0 \quad\left(r_{i}^{\prime} \in S_{Q}\right) .
$$

By the linear independency of the leading forms of $1, e_{Q}, f_{Q}, e_{Q} f_{Q}$ over $S_{Q}$, $r_{i}^{\prime}=0$ for all $i$. Then by the nature of these $r_{i}^{\prime}$, we see that $r_{4}=0$, hence $r_{2}=$ $r_{3}=0$. We conclude $r_{i}=0 \forall i$. End of lemma.

Lemma 2.4. $R\left[d_{1}, \cdots, d_{k}, \cdots\right]=\left\{\left(v_{1}+v_{2} d\right) / x^{l} \mid v_{i} \in R, l \geq 0\right\} \cap R^{*}$.

Proof. If $\left(v_{1}+v_{2} d\right) / x^{l}=r^{*} \in R^{*}$ where $v_{i} \in R$ and $l \geq 1$ then $x^{l} r^{*}-v_{2} d_{l} x^{l}=$ $v_{1}+\alpha_{l} v_{2}$. It follows since $R$ is normal and $R^{*}$ is integral over $R$ that

$$
v_{1}+v_{2} \alpha_{l}=x^{l} \cdot v \quad \text { where } v \in R \text {. }
$$

Thus we get $r^{*}=v+v_{2} d_{l} \in R\left[d_{1}, d_{2}, \cdots, d_{k}, \cdots\right]$.

If $b \in R\left[d_{1}, d_{2}, \cdots, d_{k}, \cdots\right]$ then $b \in R^{*}$ and $\exists k$ such that $b \in R\left[d_{k}\right]$ as $R\left[d_{1}, d_{2}, \cdots, d_{p}\right]=R\left[d_{p}\right]$. Thus $b=v_{1}+v_{2} d_{k}, v_{i} \in R$. Thus

$$
b=\left[\left(x^{k} v_{1}+v_{2} \alpha_{k}\right)+v_{2} d\right] / x^{k} .
$$

Thus $b \in\left\{\left(v_{1}+v_{2} d\right) / x \mid v_{i} \in R, l \geq 0\right\} \cap R^{*}$. End of lemma.

Proposition 2. $R\left[d_{1}, d_{2}, \cdots, d_{k}, \cdots\right]=T$.

Proof.

C: $T$ normal, and $R^{*}$ integrally closed and integral over $R$ imply $R^{*} \cap$ quotient field $R[d]=T$. Since $d_{N}=\left(d+a_{N}\right) / x^{N}, N=1,2, \cdots, d_{N} \in R^{*} \cap$ quotient field $R[d]$. Thus $d_{N} \in T$.

ㄹ: Let $b \in T$. By Lemma 2.1, $\exists k$ such that $b \in R\left[e_{1}, f_{1}, \cdots, e_{k}, f_{k}\right]$. Thus $\exists N$ such that $x^{N} b \in R\left[e_{1}, f_{1}\right]$. So $x^{N} b=a_{0}+a_{1} e_{1}+a_{2} f_{1}+a_{3} e_{1} f_{1}$ where $a_{i} \in R$. Also $x^{N} b=a+a^{\prime} d$ where $a, a^{\prime} \in$ the quotient field of $R$. Thus by Lemma 2.3,

$$
a=a_{0}, \quad a^{\prime} y x=a_{1}, \quad a^{\prime} z x=a_{2} \text { and } 0=a_{3} .
$$

By Theorem $1, R$ is a UFD so we can write $a^{\prime}=w_{1} / w_{2},\left(w_{1}, w_{2}\right)=1 ; w_{i} \in R$. Then $y$ and $z$ being distinct primes of $R$ allow us to conclude from $(*)$ that $w_{2}$ divides $x$ in $R$. Thus

$$
x^{N+1} b=x a_{0}+\left(x w_{2}^{-1}\right) w_{1} d \in R[d] .
$$

Thus by Lemma $2.4, b \in R\left[d_{1}, \cdots, d_{k}, \cdots\right]$. End of Proposition 2 .

Let $N>1$; we are to prove that $I_{N}$ is a UFD. But first some lemmas.

Lemma 3.1. $H_{N}$ is a UFD. 
Proof. Follows from Lemma 2.2.

Lemma 3.2. $\left[K_{N}: L_{N}\right]=2$.

Proof. $f_{N} \in L_{N}\left[e_{N}\right]$ and $H_{N}=I_{N}\left[f_{N}, e_{N}\right]$ implies $L_{N}\left[e_{N}\right]=K_{N}$. Thus $e_{N}$ being square integral over $L_{N}$ implies $\left[K_{N}: L_{N}\right]=2$, as $K_{N} \neq L_{N}$.

Thus $\left\{e_{N}\right\}$ is a 2-base for $K_{N}$ over $L_{N}$. Define the following $L_{N}$-derivation of $K_{N}: \Delta\left(e_{N}\right)=z f_{N}$.

Lemma 3.3. $\operatorname{Ker}(\Delta)=L_{N}$ and $\Delta\left(H_{N}\right) \subseteq H_{N}$.

Proof. $K_{N} \supseteq \operatorname{Ker}(\Delta) \supseteq L_{N}$ and Lemma 3.2 imply $\operatorname{Ker}(\Delta)=L_{N^{*}} \Delta\left(H_{N}\right) \subseteq H_{N}$ is easily verified by checking the action of $\Delta$ on $f_{N}$ and $e_{N} f_{N}$.

Lemma 3.4. $I_{N}$ is integrally closed.

Proof. Let $b \in$ integral closure of $I_{N}$. By Lemma 3.1, $b \in H_{N}$, so

$$
b=t_{1}+t_{2} e_{N}+t_{3} f_{N}+t_{4} e_{N} f_{N^{\prime}} \quad t_{i} \in S_{N^{*}}
$$

Since $b \in L_{N}$,

$$
b=a+a^{\prime} d_{N}, \quad a, a^{\prime} \in \text { the quotient field of } S_{N^{*}}
$$

By Lemma 2.3, $t_{1}=a, t_{2}=y a^{\prime}, t_{3}=z a^{\prime}, t_{4}=0$. Letting $a^{\prime}=w / v$, where $w, v \epsilon$ $S_{N}$, we have $t_{2} v=w y$ and $t_{3} v=w z$. Since $S_{N}$ is a UFD in which $y$ and $z$ are relatively prime, we get $v$ divides $w$ in $S_{N}$. Thus $b=t_{1}+a^{\prime} d_{N}, t_{1}, a^{\prime} \in S_{N}$. Thus $b \in I_{N}$ and $I_{N}$ is integrally closed.

Lemma 3.5. $\operatorname{Ker}(\Delta) \cap H_{N}=I_{N}$.

Proof. Follows from Lemmas 3.3, 3.4 and $H_{N}$ being integral over $I_{N}$.

Lemma 3.6. $\Delta^{2}=y \Delta$.

Proof. Follows from an easy calculation; see Lemma A.

From here on $D$ denotes the logarithmic derivatives of $H_{N}$ with respect to $\Delta$. $D^{\prime}$ denotes the logarithmic derivatives of unity of $H_{N}$ with respect to $\Delta$. (See Theorem 3.)

Lemma 3.7. Let $t \in D$. Then if $t$ is a unit of $H_{N^{\prime}} t \in D^{\prime}$.

Proof. By Lemmas B, 3.1, 3.2 and 3.6, $\Delta t=t^{2}+y t$. As $y \in \operatorname{Ker} \Delta$, $\Delta(t+y) /(t+y)=t$. As $t+y$ is a unit of $H_{N}, t \in D^{\prime}$.

Lemma 3.8. $\left(D \cap(z, y) H_{N}\right) \cup\left(D \cap \operatorname{Units}\left(H_{N}\right)\right)=D$.

Proof. Let $b \in D$. We shall show if $b \notin \operatorname{Units}\left(H_{N}\right)$, then $b \in(z, y) H_{N}$. Assume then that $b \in D \backslash$ Units $\left(H_{N}\right)$. Then

$$
b=v_{1}+v_{2} e_{N}+v_{3} f_{N}+v_{4} e_{N} f_{N^{\prime}} \quad v_{i} \in S_{N^{*}}
$$


As in Lemma 3.7, $\Delta(b)=y b+b^{2}$. This is equivalent to, by Lemma 2.3,

$$
v_{4} z f_{N}^{2}+y v_{1}=v_{1}^{2}+v_{3}^{2} f_{N}^{2}+v_{4}^{2} e_{N}^{2} f_{N^{\prime}}^{2}, \quad v_{2}=0 .
$$

By an easy reasoning, none of the $v_{i}$ are regular in $x$. Also, as $b$ is a nonunit in $H_{N}$, it is a nonunit in $R^{*}$. This follows from $q \in R^{*} \Rightarrow q^{2} \in H_{N}$. Thus we conclude $v_{i} \in(z, y) R^{*}$.

We finally conclude $b \in(Z, Y) H_{N}$ by the following

Lemma 3.8.1. $(z, y) R^{*} \cap S_{N} \subseteq(z, y) S_{N^{*}}$

Proof. Let $\alpha \in(z, y) R^{*} \cap S_{N}$. Then as $S_{N}$ is a power series ring, $\alpha \epsilon$ $(z, y) S_{N}$.

Lemma 3.9. $D^{\prime} \supseteq D \cap(z, y) H_{N}$.

Proof. Let $Q=\left\{(v, w) \mid v, w \in H_{N}\right.$ and $\left.v z+w y \in D \cap(z, y) H_{N}\right\}$. Define $\gamma$, $\alpha, \beta: Q \rightarrow H_{N}$ as follows:

Let $\left(r^{\prime}, s^{\prime}\right) \in Q$ and let $t=r^{\prime} z+s^{\prime} y$. Then

$$
t=r \Delta e_{N}+s \Delta f_{N} \quad \text { where } r=r^{\prime} / f_{N^{\prime}}, \quad s=s^{\prime} / f_{N} .
$$

By Lemma B and the proof of Lemma 3.7, it follows that

$$
\left(\Delta r+r^{2} \Delta e_{N}\right) \Delta e_{N}=\left(\Delta s+s^{2} \Delta f_{N}\right) \Delta f_{N}
$$

Since $\Delta e_{N}, \Delta f_{N}$ are relatively prime in $H_{N}$, a UFD by Lemma 3.1, $\exists$ unique $b \epsilon$ $H_{N}$ such that

$$
\Delta s=s^{2} \Delta f_{\mathrm{N}}+b \Delta e_{N}
$$

By derivations of (1), using Lemma 3.6, one gets $\Delta b \Delta f_{N}=0$, so $\Delta b=0$.

Now let $\beta\left(\left(r^{\prime}, s^{\prime}\right)\right)=1+r e_{N}+s f_{N}+(r s+b) e_{N} f_{N}$, and rewrite

$$
\beta\left(\left(r^{\prime}, s^{\prime}\right)\right)=k_{0}+k_{1} e_{N}+k_{2} f_{N}+k_{3} e_{N} f_{N^{\prime}}, \quad k_{i} \in S_{N^{\prime}}
$$

Also let

$$
\begin{aligned}
& r^{\prime}=r_{1}+r_{2} e_{N}+r_{3} f_{N}+r_{4} e_{N} f_{N}, \\
& s^{\prime}=s_{1}+s_{2} e_{N}+s_{3} f_{N}+s_{4} e_{N} f_{N}, \quad r_{i}, s_{i} \in S_{N},
\end{aligned}
$$

and let $r_{i}^{0}, s_{i}^{0}$ be the constant terms of the power series $r_{i}, s_{i}$, respectively. Define $\alpha\left(\left(r^{\prime}, s^{\prime}\right)\right)$ to be the constant term of $k_{0}$. Thus $a\left(\left(r^{\prime}, s^{\prime}\right)\right)=1+r_{4}^{0} b_{N}^{2}+s_{1}^{0}+$ $r_{1}^{0} s_{4}^{0} b_{N}^{2}+r_{2}^{0} s_{3}^{0} b_{N}^{2}+r_{3}^{0} s_{2}^{0} b_{N}^{2}+r_{4}^{0} s_{1}^{0} b_{N}^{2}$ (we have used Lemma 3.5 and that $\Delta b=0$ ) and define $\gamma\left(\left(r^{\prime}, s^{\prime}\right)\right)=1+b_{N}^{2} r_{4}^{0}$. Now let $t_{0} \in D \cap(z, y) H_{N}, t_{0}=r^{\prime} z+s^{\prime} y$. One has

$$
\Delta \beta\left(\left(r^{\prime}, s^{\prime}\right)\right)=\beta\left(\left(r^{\prime}, s^{\prime}\right)\right) t_{0} .
$$


One would like to have $\beta\left(\left(r^{\prime}, s^{\prime}\right)\right)$ a unit, for then $t_{0} \in D^{\prime}$, but this is not necessarily so. However, we have $\alpha\left(\left(r^{\prime}, s^{\prime}\right)\right) \neq 0$ implies $\beta\left(\left(r^{\prime}, s^{\prime}\right)\right)$ is a unit by the reasoning contained in Lemma 2.2. So suppose $\alpha\left(\left(r^{\prime}, s^{\prime}\right)\right)=0$.

Case I. Assume $\gamma\left(\left(r^{\prime}, s^{\prime}\right)\right) \neq 0$. Then $\alpha\left(\left(r^{\prime}, s^{\prime}+1\right)\right) \neq 0$. So if $t^{\prime}=r^{\prime} z+$ $\left(s^{\prime}+1\right) y$ then $t^{\prime} \in D \cap(Z, Y) H_{N}$ using Lemmas B and 3.6. So $\beta\left(\left(r^{\prime}, s^{\prime}+1\right)\right)$ is a unit. As $\Delta \beta\left(\left(r^{\prime}, s^{\prime}+1\right)\right)=\beta\left(\left(r^{\prime}, s^{\prime}+1\right)\right) t^{\prime}$, then

$$
\Delta\left(\beta\left(\left(r^{\prime}, s^{\prime}+1\right)\right) \cdot f_{N}\right)=\beta\left(\left(r^{\prime}, s^{\prime}+1\right)\right) \cdot f_{N} \cdot t_{0}
$$

and $\beta\left(\left(r^{\prime}, s^{\prime}+1\right)\right) \cdot f_{N}$ is a unit. Thus $t_{0} \in D^{\prime}$.

Case II. Assume $\gamma\left(\left(r^{\prime}, s^{\prime}\right)\right)=0$. Let $t^{\prime}=\left(r^{\prime}+e_{N} f_{N} / e_{N}^{2}\right) z+s^{\prime} y$. Then $t^{\prime} \epsilon$ $D \cap(Z, Y) H_{N}$, using Lemmas B and 3.6. Note that as the constant term of $1 / e_{N}^{2}$ is $1 / b_{N}^{2}$,

$$
\gamma\left(\left(r^{\prime}+e_{N} f_{N}^{\prime} e_{N}^{2}, s^{\prime}\right)\right) \neq 0 .
$$

Thus since either $a\left(\left(r^{\prime}+e_{N} f_{N} / e_{N}^{2}, s^{\prime}\right)\right) \neq 0$ or, by Case I, $\exists$ a unit $u \in H$ such that $\Delta u=u t^{\prime}$, then $\Delta\left(e_{N} u\right)=e_{N} u t_{0}$ and $e_{N} u$ is a unit. Thus $t_{0} \in D^{\prime}$. End of lemma.

Lemma 3.10. $D^{\prime}=D$.

Proof. Follows from Lemmas 3.7, 3.8, 3.9.

Proposition 3. $I_{N}$ is a UFD.

Proof. Follows from Theorem 3 and Lemmas 3.1, 3.3, 3.5 and 3.10.

Remark. In proving Lemma 3.9, some techniques of P. Samuel were used that are found in Lemma 3 of [3].

Proposition 4. T is a UFD.

Proof. In view of Proposition 1, we need only show the minimal primes of $T$ are prinicipal.

Let $P$ denote a minimal prime of $T$. By Proposition $1, \exists a \in T$ such that $P T P=\alpha \cdot T P$. As $d_{i} \in I_{j}, i \leq j$, and $T=R\left[d_{1}, d_{2}, \cdots, d_{k}, \cdots\right]$ (Proposition 2), we see that $\exists M$ such that $\alpha \in I_{M} \subseteq T$. Since $T$ is an integral extension of $I_{M}$, and by Lemma 3.4, $P^{\prime}=P \cap I_{M}$ is a minimal prime of $I_{M}$. Thus, by Proposition $3, P^{\prime}$ is principal. Let $\beta$ generate $P^{\prime}$. Since the squares of elements of $T$ lie in $I_{M}$, no two primes can contract to the same prime in $I_{M}$. Thus $\beta$ is not contained in any other minimal prime of $T$.

As $\alpha \in P^{\prime}, \quad \exists q \in T$ such that $\alpha=q \cdot \beta$. Thus $\beta \cdot T Q=P T Q$, for every minimal prime $Q$, of $T$.

By the following lemma, $\beta$ generates $P$. 
Lemma 4.1. Let $R$ be a Krull domain such that $\beta, \delta \in R$. Let $\beta$ divide $\delta$ in $R_{p} \forall$ minimal prime $P$. Then $\beta$ divides $\delta$ in $R$.

Proof. As $(\beta R: \partial R)$ is divisorial, $(\beta R: \delta R) \cap R$ is such. As $(\beta R: \delta R) \cap R$ is integral, it must contain 1 or be contained in some height 1 prime. As the latter is not possible, $1 \in(\beta R: \partial R)$. Thus $\beta$ divides $\delta$ in $R$. (See [3, pp. 1, 7].)

We are now able to state

Theorem. Let $K$ be a field of characteristic two such that $\left[K: K^{2}\right]$ is countably infinite. Let $\left\{b_{i}, c_{i}\right\}_{i}^{\infty}=1$ be a two-base for $K$ over $K^{2}$. Let $R=$ $K^{2}[[x, y, z]][K]$,

$$
d=\sum_{i=1}^{\infty} b_{i} y x^{i}+c_{i} z x^{i} .
$$

Then $T=$ the integral closure of $R[d]$ is a three dimensional non-Noetherian quasi-local factorial ring.

\section{REFERENCES}

1. M. Nagata, Local rings, Interscience, New York, 1962. MR $27 \# 5790$.

2. P. Samuel, Classes de diviseurs et dérivées logarithmiques, Topology 3 (1964), suppl. 1, 81-96. MR $29 \# 3490$.

3. - Lectures on unique factorization domains, Lectures in Math., no. 30, Tata Institute of Fundamental Research, Bombay, 1964. MR 35 \#5428.

DEPARTMENT OF MATHEMATICS, UNIVERSITY OF ROCHESTER, ROCHESTER, NEW YORK 14627 